\title{
Special Issues for Pregnant Women with Mental Illness
}

Heather Gilbert ${ }^{*}$, Caroline Gurvich and Jayashri Kulkarni

The Monash Alfred Psychiatry research centre (MAPrc), Central Clinical School, Faculty of Medicine, Nursing and Health Sciences, Monash University \& The Alfred Hospital, Melbourne, Australia

*Corresponding author: Heather Gilbert, The Monash Alfred Psychiatry research centre (MAPrc), Central Clinical School, Faculty of Medicine, Nursing and Health Sciences, Monash University \& The Alfred Hospital, Melbourne, Australia, MAPrc, Level 4, 607 St Kilda Rd, Melbourne, Victoria, 3004, Tel: (03) 9076-6591; Fax: (03) 9076-6588; E-mail: heather.gilbert@monash.edu

Received date: May 29, 2015, Accepted date: July 04, 2015, Published date: July 12, 2015

Copyright: (C) 2015 Gilbert $\mathrm{H}$ et al. This is an open-access article distributed under the terms of the Creative Commons Attribution License, which permits unrestricted use, distribution, and reproduction in any medium, provided the original author and source are credited.

\section{Abstract}

Background: Women who have a mental illness are no less likely to plan for motherhood than any other group in the community. On the contrary, many women with mental illnesses are fully functioning members of society, juggling employment, career, study, family and parenting in conjunction with the 'normal' stressors of life. However this is not the case for all. Their burden of illness places extra strain and stress on their lifestyle and family dynamics, often being exacerbated during pregnancy and following the birth. Although some of these effects may be ameliorated using different treatment modalities, they are frequently offered in a disconnected fashion. The introduction of an integrated, holistic approach, as the primary model of care, can successfully incorporate aspects of assessment, prevention and management, thereby strengthening maternal mental health in pregnancy and encouraging healthy mother and infant outcomes.

Purpose: This paper will highlight and discuss special issues for pregnant women with mental illnesses, to increase clinical awareness, encourage risk assessment and promote management planning, by using an integrated model of care in support of women during pregnancy and in early motherhood.

Conclusion: Pregnancy places extraordinary stress on every conceivable aspect of a woman's life, including the exponential changes to her own body, intimate relationships, family groupings, career development and lifestyle adjustments. There is true, ongoing, holistic change to the extent that mental health can be compromised. Knowledge of such changes and the opportunity to encourage healthy outcomes must, therefore, be fully supported with an integrated approach by clinicians, consumers and carers.

Keywords: Pregnancy; Perinatal mental illness; High risk; Integrated care; Mother and Infant outcomes

\section{Introduction}

Pregnancy is a time of great joy for many women, however it can also be a time of considerable challenge - physical, biological and emotional [1]. This includes having a mental illness for a minority of women, who, nevertheless, wish to raise families and be active members of their family groups. In this group's situation, mental illness, pregnancy and motherhood form a hazardous triangle of possible adverse perinatal events.

The inherent biological changes in perinatal hormones, neurotransmitters and neurosteroids impact significantly on mental illness in the pregnant woman and new mother, creating vulnerability to depression and psychosis [2]. Furthermore, the effect of deinstitutionalisation on mental health care and the advance of second generation, or atypical, antipsychotic medication use has seen a rise in the prevalence of pregnancies in women with mental disorders $[3,4]$.

Conjointly, acknowledgment and understanding of perinatal mental illness and maternal need, amongst clinicians, consumers, carers and families can be lacking, but is an essential part of encouraging healthy mother-infant outcomes, particularly as maternal suicide is significant in western societies $[1,5]$.
Current treatments include psychotropic medications, psychotherapeutic interactions with trained clinicians, community engagement programmes, parenting programmes and the development of robust support networks which endeavour to ensure that women access available services to meet their needs $[2,6]$.

Integrated models of care which focus on the needs of women with perinatal mental illness are patchy, [7], even though guidelines for clinical practice have been available for some time, for example, in Australia (the Beyondblue Guidelines) [8], and in the United Kingdom, (NICE - the National Institute for Health and Care Excellence - Antenatal and postnatal mental health guidelines) [9]. Unfortunately this does not always equate to action in the clinical sense, for a variety of reasons, including lack of awareness, agreement difficulties, querying the efficacy of guideline and self, attitudes towards the guideline and outcome expectations [10]. Poor outcomes affect the adherence to guidelines, resulting in sporadic assessments, intervention and treatment plans and therefore an ongoing lack of integrated care between the disciplines, all adversely affecting the mother and infant.

The following discussion will begin with a general review of the impact of perinatal mental illness, followed by specific antenatal issues and early motherhood problems. The prevalence, risk factors, assessment and management, pharmacological treatments, specific needs and experiences of women, and nursing implications are all considered in the development of an integrated model of care. 


\section{Overview and Prevalence of Perinatal Mental Illness}

The overall lifetime prevalence of mental illness is reportedly $29 \%$, with women experiencing more mood and anxiety disorders, while substance use disorders are more common in men [11]. Furthermore, mood and anxiety disorders are also more prevalent in pregnancy [12]. Women with a history of mood or psychotic disorders are in the high risk category for relapse post-birth, placing relapse prevention at the top of the priority list [13].

Perinatal mental illness is known to occur during pregnancy, following the birth and up to three years postpartum [14,15]. This includes mood, anxiety and psychotic disorders, which can present as a relapse of pre-existing illness or as a de novo event [16]. There is an estimated $40 \%$ of women who experience some form of psychological adversity during pregnancy or following the birth, with approximately $10-15 \%$ of this group diagnosed with a mental illness [12,17]. This knowledge highlights the necessity to support holistic, integrated, woman-centred care, with achievable health benefits in terms of service access, lifestyle factors, pharmacological therapies and improved mother-infant outcomes, including attachment. However, although the future for children of mothers with mental illness is currently uncertain, there appears to be an increased risk of offspring developing mental illness in later life $[1,4,16-18]$.

Mental illness during pregnancy, birth and the postpartum period can present in many different ways, each with its own characteristics but all requiring careful clinical consultation, planning and management, in close collaboration with women and their families.

\section{Mood disorders}

Perinatal mood disorders include depression and bipolar affective disorder (BPAD), which are usually non-psychotic in nature [17]. Schizoaffective disorder is a combination of both schizophrenia and mood disorder, being either bipolar type or depressive type, and can therefore be difficult to diagnose accurately with the merging or blurring of boundaries $[19,20]$.

Depression commonly occurs during pregnancy and/or following the birth, including up to 12 months postnatally, although it can also be a relapse of a pre-existing illness [16,21]. Significantly, the rate of perinatal major and minor depression has been reported between $5-20 \%$ in high income countries [22-26] while low income countries have a reported incidence of $15-20 \%$ [15,27]. Furthermore, antenatal depression alone is known to be both common and significant, with an estimated prevalence of $10-20 \%$ [28,29]. Depressive symptoms include low mood, anhedonia, amotivation, feelings of numbness, tearfulness, loss of appetite, insomnia (although difficult to assess at times with a new infant to care for), difficulty making decisions and harmful thoughts towards self or baby.

'Baby blues', or 'postnatal blues', experienced by approximately $50-85 \%$ of women following the birth, are generally transient, manifesting by day 4-5 post-birth, approximately when full milk flow is established. The symptoms of mild depression, anxiety, mood changes and tearfulness, tend to resolve spontaneously by day 10-12 $[16,17]$.

Postnatal depression, however, is a continual, ongoing, decline of mood and depressive nature, to the extent that it significantly affects maternal functioning, wellbeing and interaction with the infant, family and clinicians. Medication treatment generally consists of antidepressants. It is suggested that maternal perinatal depression, often associated with stress and anxiety, contributes to poor pregnancy, birth and postpartum outcomes for both mother and infant, whilst also affecting parenting skills and mother-infant attachment [14]. Further, lack of attention to the skills of daily living, including nutrition, poor antenatal clinic attendance and an increase in smoking, alcohol and illicit drug use are not uncommon in this group [28]. Antidepressants are generally prescribed, for example, sertraline (Zoloft), escitalopram (Lexapro), venlafaxine (Effexor).

Bipolar affective disorder (BPAD) has a life time prevalence of $\geq 1 \%$ [30]. It is characterised by either manic, depressive or mixed episodes [2], some presenting de novo following the birth, while approximately $50 \%$ of women with a history of BPAD will experience a relapse postpartum [16,31,32]. Typical manic symptoms include elevated mood, verbosity, hyperactivity, imprudent spending, grandiosity and disinhibited behaviours along with increased libido, often leading to sexual promiscuity [2]. The illness can cycle rapidly, often between mania and depression, in tandem with gonadal hormonal shifts $[33,34]$. Apart from possible mood swings and medication noncompliance, maternal pregnancy complications include antenatal haemorrhage, while there is a reported incidence of small for gestational age (SGA), intrauterine growth retardation (IUGR) and premature birth in infants $[14,35]$. Medication treatments consist of mood stabilisers and/or antipsychotics, but can be difficult to manage effectively [2]. Mood stabilisers include lithium, lamotrigine (Lamictal), carbamazepine (Tegretol) and sodium valproate (Epilim), while antipsychotics include typical, (first generation antipsychotics) and atypical (second generation antipsychotics), as discussed in the section on 'Pharmacological treatments for perinatal mental illness'.

\section{Anxiety disorders}

Perinatal anxiety disorders, which typically having their onset in the early childbearing years, [36], are thought to be more common than generally acknowledged and therefore may be missed in diagnosis [15]. They include generalised anxiety disorder (GAD), panic disorder, obsessive compulsive disorder (OCD), post-traumatic stress disorder (PTSD) and certain phobias, such as tokophobia (pathological fear of pregnancy and childbirth), which is beginning to receive more clinical attention, particularly with regard to increased requests for caesarean section or the avoidance of pregnancy altogether $[16,26]$.

Generalised anxiety disorder (GAD) presents as excessive worry that interferes with a wide range of normal daily living activities, often with no apparent trigger present.

Panic disorder, whose symptoms include shortness of breath, dizziness and palpitations, has a rapid, unpredictable onset caused by extreme worry, which is often related to the fear of further panic attacks.

Obsessive compulsive disorder (OCD) describes behaviours which are repetitive (compulsions) and thoughts which are intrusive (obsessions). Obsessions create anxiety which can subsequently be relieved by compulsive behaviour. In the perinatal context, obsessions frequently include thoughts of harming the fetus or infant, whether that be intentional or not [37]. Women who experience these thoughts are often further isolated by the stigma that surrounds mental illness as a whole, which in turn prevents them from reporting such thoughts to their health care providers [38].

Post-traumatic stress disorder (PTSD) is a fearful or helpless response to an experienced traumatic event, as in a threat or injury to self or others [36]. 
Between $4.5-15 \%$ of women experience an anxiety disorder perinatally, including PTSD while pregnant and/or following the birth $[15,16,39]$. Anxiety disorders range from extreme worry to panic attacks and can occur both during and after pregnancy [26]. It is also thought that for women with postnatal depression, approximately $66 \%$ will experience a comorbid anxiety disorder $[15,26,40]$. Medications include benzodiazepines, such as alprazolam (Xanax), diazepam (Valium), lorazepam (Ativan); beta blockers, for example, propranolol (Inderal), atenolol (Tenormin); and anti-depressants.

\section{Psychotic disorders}

Psychotic disorders, such as schizophrenia, present manifold risk factors for mother and infant both during and following pregnancy, including the possibility of maternal antenatal mental health deterioration [4]. Symptoms include delusions, hallucinations, grandiosity (positive), blunted affect, emotional withdrawal, stereotyped thinking (negative) anxiety, depression, lack of insight (general) [41]. There is approximately a $1-2 \%$ chance of developing schizophrenia over the lifespan [16,42], while the risk of concurrent mental illness following the birth, in women who already have schizophrenia, particularly during the first 3 months, is significant $[16,43,44]$. These factors, combined with poor psychosocial behaviours, can contribute to sub-optimal outcomes for mother and infant. Risk factors for infants include small for gestational age (SGA), intrauterine growth retardation (IUGR), prematurity and APGAR scores of $\leq 7$ [14], the latter translating to symptoms of poor respiratory function, bradycardia, hypoxia and hypotonia [45]. Infants of mothers in this group are also more likely to be affected by poor mother-infant attachment, bonding, and foster care placement [14]. Medication includes typical (first generation) or atypical (second generation) antipsychotics, as discussed in the section on 'Pharmacological treatments for perinatal mental illness'.

Puerperal psychosis, although rare, is a severe mental illness which affects 1-2:1000 mothers, usually within the first 2 weeks post-birth $[14,16,46-49]$. Up to $60 \%$ of women who suffered puerperal psychosis with their first pregnancy are at risk of psychosis relapse with subsequent pregnancies [16,50]. Comorbid bipolar affective disorder can be part of puerperal psychosis $[14,16,49,51]$. In addition, there is a strong hereditary link, with an overall recurrence rate of one in four pregnancies (46). Concerningly, in this group there is an estimated 1:500 women who not only have suicidal ideation, plan and intent, but who actually commit suicide [52]. Furthermore, there is a known link between infanticide and suicide, with a reported $16-29 \%$ of mothers who commit infanticide [53].

\section{Impact of Perinatal Mental Illness}

Pregnancy in women with mental disorders is associated with more risks for the woman and her unborn infant compared with mentally well women. Sub-optimal obstetric outcomes have been described for the mother and infant [16]. Maternal causes include deterioration in mental health status, which is often due to hormonal changes, medication maladjustment or cessation, metabolic disorders, intrapartum complications, emergency birth procedures, such as increased rates of caesarean births, and serious adverse events following the birth, such as postpartum haemorrhage. In addition, caesarean section constitutes major surgery, which brings its own set of risks (airway management, haemorrhage, wound infection), particularly for women with multiple co-morbidities, including prepregnancy obesity and gestational diabetes mellitus (GDM). These scenarios represent a potentially traumatic response to unexpected adverse events, which can exacerbate current mental illnesses or initiate a de novo episode, for example, post-traumatic stress disorder (PTSD) or depression.

Fetal risks comprise premature birth, intra-uterine growth restriction (IUGR), small for gestational age (SGA) at birth, respiratory distress, neonatal abstinence syndrome (medication withdrawal), congenital anomalies and infant developmental delay [16]. Maternal perinatal mental ill health can also be exacerbated by lifestyle stressors and psychosocial factors. These include coming from a low socio-economic background, having poor self-care, poor education and poor nutrition, smoking, alcohol and illicit drug use and abuse, lack of reliable supports, isolation in the community, whether that be urban or rural, family violence, intermittent/no access to available health services, lack of contraception and family planning advice, indiscriminate sexual behaviours and decisions, social stigma and vulnerability [4].

Of further concern, approximately $50 \%$ of pregnancies are unplanned, across all population groups [5], while many women, particularly those with chronic mental illness, for example schizophrenia, become single mothers [2,54]. In addition, and increasing the likelihood of unplanned pregnancies, sexual activity amongst teenagers, in general, has increased significantly over the last 50 years [55], with the age for first sexual encounters decreasing. Women with mental illness are particularly vulnerable to sexually explicit behaviour or unwanted sexual activity, often with a history of poor decision-making and pressured, violent relationships.

\section{Risk Factors for Perinatal Mental Illness}

\section{Maternal risk factors (affecting mother and infant)}

Perinatal mental illness can affect any woman, regardless of her socio-economic status, including women who do not present initially as being at risk of developing mental ill health. Lack of screening before, during and after pregnancy can miss those who might otherwise have been identified as being at risk [56]. Maternal risk factors for perinatal mental illness, as mentioned, can therefore relate to a de novo event, a current and ongoing mental illness or exacerbation of a current mental illness. Risk factors include socioeconomic problems, especially a history of family violence. There is often violence in their own childhood, which can have far-reaching effects on the woman's ability to parent a new infant [57]. Furthermore, partner-related violence is reported to increase during pregnancy [58] and to be associated with perinatal post-traumatic stress disorder (PTSD), depression and anxiety [36,59]. Other predisposing factors for perinatal mental illness include incomplete schooling, poor decision making skills, (which can lead to poor choice of partner), smoking, alcohol and illicit drug use, difficulty managing a pre-existing mental illness, [16] cessation of medication, avoidance of clinical services, insecure, unstable housing and unmet nutritional needs with problematic perinatal impact on both mother and infant.

Maternal co-morbidities, likewise, present many challenges, often compromising fetal development and infant progress at birth. For example, pre-pregnancy obesity is closely associated with the development of gestational diabetes mellitus (GDM), which carries a significant occurrence risk of 1-3\%, across all pregnancies [60]. Although GDM generally resolves post-birth, approximately $50 \%$ of women with GDM will develop diabetes mellitus (DM) Type 2 in later 
life [61]. There is also thought to be a strong genetic link with familial diabetes mellitus and GDM in first degree relatives [62,63]. Maternal complications linked with GDM include hypertension, pre-eclampsia and early induction of labour at approximately 38 weeks gestation, which often involves an elective caesarean section due to potential adverse neonatal outcomes.

Neonatal risk factors and anomalies include premature birth, macrosomia, respiratory distress, sub-optimal placental function, APGAR $\leq 7$, Neonatal Intensive Care Unit (NICU) admission, birth trauma, shoulder dystocia and hypoglycaemia [60,64]. Furthermore, these risk factors may be worsened by the use of maternal medications in the treatment of perinatal mental illness, particularly antipsychotics, which have a known effect on maternal weight gain and the development of GDM in pregnancy $[63,65,66]$.

\section{Infant risk factors (affecting infant and mother)}

The subsequent impact of maternal risk factors on infant outcomes is considerable, as described in relation to maternal gestational diabetes mellitus (GDM). Other factors that can influence suboptimal infant outcomes as a result of maternal mental illness include premature birth, small for gestational age (SGA), intrauterine growth retardation (IUGR), emergency caesarean due to maternal anxiety, relapse of illness or intrapartum and comorbid complications, neonatal respiratory distress syndrome, medication withdrawal and frequent admission to either a Special Care Nursery (SCN) or Neonatal Intensive Care Unit (NICU), or both, or indeed a Mother/ baby Unit admission for deteriorating maternal mental health [6] [63]. Additionally, poor mother-infant attachment and neglect of self and the infant increases the risk of the infant subsequently developing a mental illness in adolescence or adulthood. Hence the accumulation of maternal life stressors, particularly where mental illness is significant, can impact heavily on parenting style and skill, at times resulting in dysfunctional families, poverty, extreme risk-taking behaviours, violence in the home and foster care arrangements for children.

Poor birth and infant outcomes can also contribute to a deterioration of maternal mental health, for example, the need for an emergency caesarean, the prospect of a painful birth [36] or the unexpected admission of the infant to the Special Care Nursery (SCN) or Neonatal Intensive Care Unit (NICU). This is also the case for women who experience infertility, miscarriage or stillbirth [36]. Such traumatic events can predispose to the development of, or resurgence of symptoms for, post-traumatic stress disorder (PTSD) [36] or depression.

\section{Stigma and Mental Illness}

The negative impacts of mental illness can be far reaching, however the biological and psychosocial aspects are further exacerbated by stigma-associated behaviours [38,67]. Women with mental illnesses can be alienated, isolated and detached from society [8] and appropriate clinical care, because of negative comments, attitudes or actions of friends, family, work colleagues and the public at large, or because their own healthcare team belittle them or maintain low expectations of their future improvement. Unfortunately, this encourages an ongoing, downward spiral of self-deprecation and selfstigmatisation. In addition, many women are not clinically assessed for perinatal mental illness, putting them further at risk. Consequently, personal and clinical relationships break down, with women drifting away from essential service access. The cycle continues with further mental health deterioration and ongoing stigma [38].

\section{Risk Assessment, Management and Care Planning}

Assessing risks and planning for pregnancy, birth and early motherhood are essential elements in the care and management of all pregnant women, but even more so for women with mental illness. At the very least, risk assessment should be initiated in the preconception stage, with consistent follow up in pregnancy, at the birth and for the first 12 months following the birth. A collaborative approach, across obstetric and mental health services, is needed to ensure that clinical teams work together in support of maternal and infant health.

Ongoing perinatal screening of all women, not just those with a mental illness, can alert clinicians to potential problem areas, as previously discussed, while at the same time putting strategies in place to address lifestyle factors, diet, mental health management, medication support, access to clinical services and comprehensive obstetric and post-birth follow up. The empowerment of a woman with mental illness involves providing her with appropriate information, encouraging her to weigh up this information and to make informed decisions together with her treating team. Forward planning of risk, management and care, are useful tools to help avoid adverse events and support those women in our communities, who are often stigmatised, vulnerable and isolated.

Current treatment modalities are many and varied, including pharmacological support, clinical supports (nurses, midwives, child and maternal health nurses, mental health nurses, general practitioners, obstetricians, paediatricians, psychologists, psychiatrists, dietitians, social workers, family planning initiatives), interpersonal psychotherapy (IPT), cognitive behavioural therapy (CBT), family therapy, parent-infant attachment support, family support services and the maintenance of robust personal support groups $[7,14]$. However, although there is clearly a wealth of expertise available, it is not always combined in an integrated approach, which results in many services being unrepresented in the total care package. This will be discussed in more detail in the section 'Achieving an integrated model of care'.

\section{Pharmacological Treatments for Perinatal Mental Illness}

Pharmacological support, in the form of psychotropic medications, includes antipsychotics, antidepressants, mood stabilisers and antianxiety preparations. Such medications are used to treat the symptoms of many mental illnesses, for example schizophrenia, bipolar affective disorder, depression and anxiety disorders. Of note, these medications are metabolised differently by women compared to men. This is due to several important factors, such as muscle/fat proportion, gastrointestinal integrity and adjunctive medication combinations [2]. Important considerations with regard to pharmacological treatments for women with perinatal mental illness include current mental state, the use of psychotropic medications at pre-conception, during pregnancy, birth and the postpartum period, the ratio of placental passage, fetal morphology and subsequent neonatal outcomes [68].

Pregnancy, therefore, requires another layer of assessment with regard to the use and safety of these medications. There is a fine balancing act between maintaining stable maternal mental health and 
the risk of fetal teratogenicity. Individual, tailored risk/benefit assessment is therefore an important part of pregnancy planning, prevention and management, bearing in mind the possibility of rapid maternal mental health deterioration, with subsequent flow-on effects to the infant, should medications be ceased.

\section{Antipsychotics}

There is some evidence that antipsychotic medication use in pregnancy is associated with a significant risk of adverse obstetric maternal and infant outcomes [69]. Even so, the effects of both typical (first generation) antipsychotics, such as chlorpromazine (Largactil), haloperidol (Haldol) and trifluoperazine Stelazine), and atypical (second generation) antipsychotics, such as quetiapine (Seroquel), olanzapine (Zyprexa) and risperidone (Risperdal), taken in pregnancy, are not studied well or reviewed in detail in the current literature. However, there is a great need to ensure stable perinatal mental health, which often means maintaining and/or adjusting medication regimes for many women, during pregnancy, in the postnatal period and in the longer term.

The Australian National Register of Antipsychotic Medication in Pregnancy (NRAMP), is a unique, descriptive, longitudinal, Australiawide research study which follows the progress of women with mental illness who take antipsychotic medication in pregnancy. This project, now in its tenth year, has focussed the attention of clinicians and researchers on important maternal and infant outcomes. Analyses describe significant neonatal respiratory distress and medication withdrawal, often in conjunction with Special Care Nursery (SCN) and/or Neonatal Intensive Care Unit (NICU) admission, in women who took second generation antipsychotics in pregnancy, when compared with whole population data [63]. This vital work continues, initially following infant development for the first year of life, with plans to extend follow up to 5 years of age, being the 'gold standard' for studies involving children.

In general, the maternal side effect profile of antipsychotic medication use includes maternal weight gain, often compounded by pre-pregnancy overweight or obesity, plus familial diabetes mellitus (DM) and gestational diabetes mellitus (GDM), hyperlipidaemia, extra pyramidal side effects, sexual dysfunction and sedation [70,71]. Fetal side effects focus on respiratory distress, medication withdrawal, premature birth, congenital anomalies, intrauterine growth retardation (IUGR), small for gestational age (SGA) [69] and the risk of neural tube defects [72].

\section{Antidepressants}

Antidepressant prescribing has increased greatly in the last 20 years [73]. Approximately $10 \%$ of all women take an antidepressant, most commonly, a selective serotonin reuptake inhibitor (SSRI) in the USA, while $3 \%$ of women take an antidepressant in Europe [15]. Furthermore, women with depression who become pregnant and abruptly stop taking antidepressant medication, are at an increased risk of relapse of depression and maternal suicide [28]. Antidepressants are also prescribed in the treatment of anxiety, PTSD and eating disorders [15]. There are many different types of antidepressants, with varying mechanisms of action, but side effects commonly include sexual dysfunction, gastro-intestinal disturbances and central nervous system effects such as insomnia and sedation [74].
Neonatal adverse outcomes in infants whose mothers took antidepressants in pregnancy include, premature birth, pulmonary hypertension, low birth weight, heart defects and medication withdrawal with maternal SSRI use in the third trimester $[16,28,29]$. In addition, there is a possible link between maternal antenatal depression and episodes of postnatal depression, with a higher risk of impaired IQ level and language development in the infant [64].

\section{Mood stabilisers}

Anti-epileptic medications, such as carbamazepine, sodium valproate and lamotrigine are often also used as 'typical' mood stabilisers. In addition, there is good perinatal literature focussing on their use and effect in pregnancy $[49,75,76]$. This literature reports significant fetal teratogenetic outcomes with all mood stabilisers, particularly and most severely, with sodium valproate $[14,49,75,77]$. Caution with typical mood stabiliser use in pregnancy is needed given the reported neonatal prematurity, intrauterine growth retardation (IUGR), small for gestation age (SGA), hypoglycaemia, withdrawal symptoms, neural tube defects, microcephaly, cardiac and craniofacial defects, and sub-optimal developmental cognitive outcomes $[16,78]$. The second generation antipsychotic medications, such as quetiapine, olanzapine and risperidone, are also used for mood stabilisation. The effects of these medications have been described in the section on antipsychotics in pregnancy.

\section{Achieving an integrated model of care}

Mental illness in the pregnant woman and new mother can present the clinician with a number of biological and psychosocial issues that need management, for both the woman and her infant. A holistic, multidisciplinary approach to care makes very good sense, but is not always put into practice [17]. This is partly because such dedicated services are in the minority [46], and also because clinicians in general are unaware of their roles and do not have adequate knowledge and training to carry them out appropriately [79].

Health care providers need to be upskilled in comprehensive service provision and research activities that expand and utilise knowledge in this area. As discussed earlier, although clinical guidelines have been developed in different disciplines, they are not always successful in that they have limited effect on clinical behaviour change (10). This can be attributed to unwieldy hospital and clinic structures, lack of knowledge flow through and unexpected outcomes [10], which are useful pointers for future framework development.

Specialist perinatal mental health services, incorporating integrated models of care $[46,80]$, are an essential tool when it comes to perinatal mental health screening, risk assessment, planning, prevention, intervention, management, and support (Figure 1). In effect they are safe guarding mothers in the present day and families of the future $[7,14,79]$. They also allow for the opportunity to develop efficacious referral pathways [7] which incorporate all clinical and alternate disciplines, thereby providing mother and infant-centred holistic care. Most countries, including wealthy nations, have too few publicly funded perinatal services. The overall goal for perinatal mental health services is active prevention, timely diagnosis and efficacious intervention [46]. This includes continuity of care and the support of robust parenting skills [18]. It is also important to support partners and families of women who experience postpartum psychosis [49,81]. 
Citation: Gilbert H, Gurvich C, Kulkarni J (2015) Special Issues for Pregnant Women with Mental Illness. J Nurs Care 4: 280. doi:

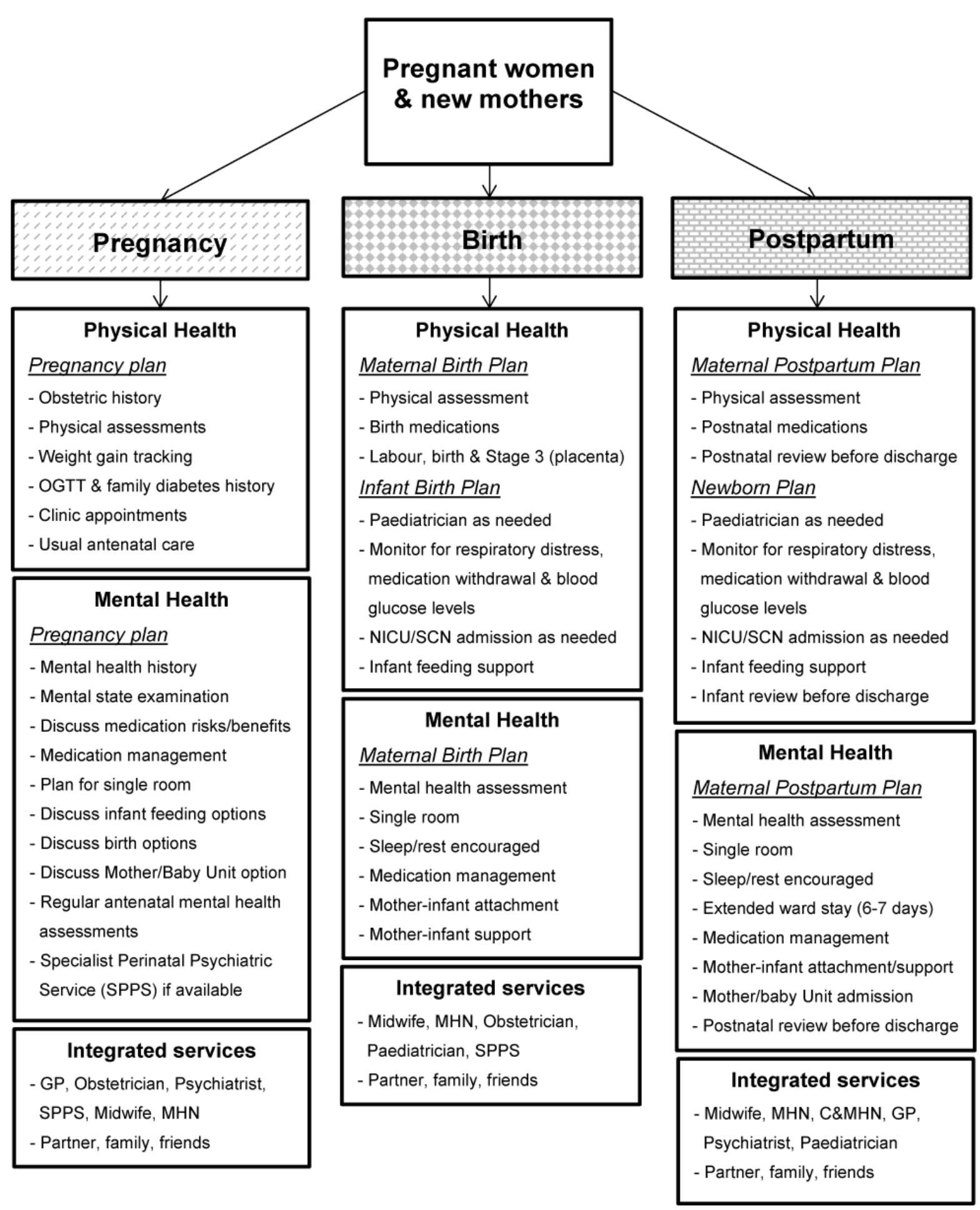

Figure 1: Integrated model of care for the screening and management of pregnant women and new mothers, SPPS $=$ Specialist Perinatal Psychiatric Service, SCN $=$ Special Care Nursery, $\mathrm{MHN}=$ Mental Health Nurse, NICU $=$ Neonatal Intensive Care Unit, C\&MHN $=$ Child \& Maternal health Nurse, OGTT = Oral Glucose Tolerance Test 


\section{The needs of women with perinatal mental illness}

What do women want in this scenario? The rights of women need to be acknowledged when making these important, life-changing decisions. When this also involves the management of mental illness during pregnancy, birth and motherhood, clinicians have a responsibility to provide appropriate care that fosters healthy outcomes. While women need to have their choices respected, they also require support, with access to appropriate information with which to make their choices $[7,79]$. A recent study has shown that pregnant women and new mothers with mental illness developed trusting relationships with their perinatal mental health worker and were then able to emulate this behaviour as robust mother-infant attachment [82]. Continuity of care and sensitivity to the needs of others are also important if women are to trust their clinicians and make good decisions based on the information with which they are provided [14]. Additionally, continuity of the clinical relationship and health information accessed were seen as fundamental parts of the whole experience during pregnancy and birth, in both urban and rural settings $[82,83]$. The antithesis of this shows that negative clinical experiences, be that physical, mental or emotional, but particularly with regard to the clinician-consumer relationship, including stigmatisation, can have long-lasting and blighted effects, at times resulting in the mother's subsequent cessation of service access, thereby putting herself and her infant at risk [82]. Furthermore, this can include the failure of clinicians to assess for, or recognise, perinatal mental illness in the first place, as discussed in the section on 'Stigma and Mental Illness'. Perinatal mental health screening and planning for pregnancy, birth and motherhood, for all women, is essential. It can be a supportive process, regardless of mental health status, ensuring that integrated services are available as needed, and has the ability to encourage women to exercise self-determination [84], empowerment, and the utilisation of help-seeking activities to support their ongoing needs across the lifespan.

\section{Nursing Implications}

This model of care encourages nurses from midwifery, mental health and general backgrounds to work together, supporting and advocating for holistic patient care. It helps to strengthen the return to basic nursing care, and supports change management which some nurses find difficult. It also allows nurses to support each other and to gain extra knowledge and skills which are transferrable into every specialty of nursing. Opportunities to teach and share with new graduates and disseminate new knowledge by way of research activities will enhance positive, healthy patient outcomes.

\section{Conclusion}

This paper acknowledges and discusses perinatal mental illness as being both complex and high risk. It also recognises that it is the clinician's responsibility to maintain safe environments, professional processes and robust practice, as discussed in the Australian Nursing and Midwifery Council report of 2007 [85], whilst also supporting the development and maintenance of infrastructure in the form of an integrated model of care which seeks to reduce the risks for mothers and infants in this vulnerable population group. Additionally, strong therapeutic relationships, comprehensive family involvement and cultural sensitivity [8] are essential to complement basic caregiving activities.
Healthy infants begin with healthy mothers who support healthy infants. This is the ultimate ongoing cycle of health benefits and outcomes that all clinicians should be aiming to achieve, in an integrated, tailored approach.

\section{References}

1. Howard LM, Piot P, Stein A (2014) No health without perinatal mental health. Lancet 384: 1723-1724.

2. Kulkarni J (2010) Special issues in managing long-term mental illness in women. Int Rev Psychiatry 22: 183-190.

3. Mowbray CT, Oyserman D, Zemencuk JK, Ross SR (1995) Motherhood for women with serious mental illness: pregnancy, childbirth, and the postpartum period. Am J Orthopsychiatry 65: 21-38.

4. Shah A, Christophersen, R (2010) Prenatal Care for Women with Schizophrenia. Current Women's Health Review 6: 28-33.

5. Cantwell R, Clutton-Brock T, Cooper G, et al (2011) Saving mothers' lives: reviewing maternal deaths to make motherhood safer: 2006-2008. The eighth report of the Confidential Enquiries into Maternal Deaths in the United Kingdom. BJOG - An International Journal of Obstetrics and Gynaecology 384: 1-203.

6. Nguyen TN, Faulkner D, Frayne JS, Allen S, Hauck YL, et al. (2013) Obstetric and neonatal outcomes of pregnant women with severe mental illness at a specialist antenatal clinic. Med J Aust 199: S26-29.

7. McNamara P (2011) Perinatal mental health. Ostetrics \& Gynaecology Magazine (RANZCOG) 13: 56-7.

8. Beyondblue (2011) Clinical Practice Guidelines: Depression and related disorders - anxiety, bipolar disorder and puerperal psychosis - in the perinatal period. A guideline for primary care health professionals. Austin M-P, Highet, $\mathrm{N}$ and the Guidelines Expert Advisory Committee (eds.), Beyond Blue, Melbourne.

9. NICE (2014) Antenatal and postnatal mental health: Clinical management and service guidance. United Kingdom: NICE. 1-62.

10. Cabana MD, Rand CS, Powe NR, Wu AW, Wilson MH, et al. (1999) Why don't physicians follow clinical practice guidelines? A framework for improvement. JAMA 282: 1458-1465.

11. Steel Z, Marnane C, Iranpour C, Chey T, Jackson JW, et al. (2014) The global prevalence of common mental disorders: a systematic review and meta-analysis 1980-2013. Int J Epidemiol 43: 476-493.

12. Fisher J, Cabral de Mello M, Patel V et al. (2012) Prevalence and determinants of common perinatal mental disorders in women in low and lower middle income countries: a systematic review. Bulletin on the World Health Organisation 90: 139-49.

13. Bergink V, Bouvy PF, Vervoort JS, Koorengevel KM, Steegers EA, et al. (2012) Prevention of postpartum psychosis and mania in women at high risk. Am J Psychiatry 169: 609-615.

14. Galbally M, Snellen M, Walker S, Permezel M (2010) Management of antipsychotic and mood stabilizer medication in pregnancy: recommendations for antenatal care. Aust N Z J Psychiatry 44: 99-108.

15. Howard LM, Molyneaux E, Dennis CL, Rochat T, Stein A, et al. (2014) Non-psychotic mental disorders in the perinatal period. Lancet 384 : 1775-1788.

16. Paschetta E, Berrisford G, Coccia F, Whitmore J, Wood AG, et al. (2014) Perinatal psychiatric disorders: an overview. Am J Obstet Gynecol 210: 501-509.

17. Mares S, Newman, L, Beulah, W (2011) Clinical Skills in Infant Mental Health: The First Three Years. Camberwell, Victoria, ACER Press, Australia.

18. Stein A, Pearson RM, Goodman SH, Rapa E, Rahman A, et al. (2014) Effects of perinatal mental disorders on the fetus and child. Lancet 384 : 1800-1819.

19. Cheniaux E, Landeira-Fernandez J, Telles L L et al. (2008) Does schizoaffective disorder really exist? A systematic review of the studies that compared schizoaffective disorder with schizophrenia or mood disorders. Journal of Affective Disorders 106: 209-17. 
20. Peralta V, Cuesta MJ (2008) Exploring the borders of the schizoaffective spectrum: a categorical and dimensional approach. J Affect Disord 108: 71-86.

21. Austin MP, Middleton PF, Highet NJ (2011) Australian mental health reform for perinatal care. Med J Aust 195: 112-113.

22. Gavin NI, Gaynes BN, Lohr KN, Meltzer-Brody S, Gartlehner G, et al. (2005) Perinatal depression: a systematic review of prevalence and incidence. Obstet Gynecol 106: 1071-1083.

23. Le Strat Y, Dubertret C, Le Foll B (2011) Prevalence and correlates of major depressive episode in pregnant and postpartum women in the United States. J Affect Disord 135: 128-138.

24. Ban L, Gibson JE, West J, Fiaschi L, Oates MR, et al. (2012) Impact of socioeconomic deprivation on maternal perinatal mental illnesses presenting to UK general practice. Br J Gen Pract 62: e671-678.

25. Berle JO (2012) Psychiatric conditions in pregnancy and postpartum. Acta Obstetrica et Gynaecologica Scandinavica 91: 38.

26. O'Hara MW, Wisner KL2 (2014) Perinatal mental illness: definition, description and aetiology. Best Pract Res Clin Obstet Gynaecol 28: 3-12.

27. Parsons CE, Young KS, Rochat TJ, Kringelbach ML, Stein A (2012) Postnatal depression and its effects on child development: a review of evidence from low- and middle-income countries. Br Med Bull 101: 57-79.

28. Udechuku A, Nguyen T, Hill R, Szego K (2010) Antidepressants in pregnancy: a systematic review. Aust N Z J Psychiatry 44: 978-996.

29. Hayes RM, Wu P, Shelton RC, Cooper WO, Dupont WD, et al. (2012) Maternal antidepressant use and adverse outcomes: a cohort study of 228,876 pregnancies. Am J Obstet Gynecol 207: 49.

30. Viguera AC, Whitfield T, Baldessarini RJ, Newport DJ, Stowe Z, et al. (2007) Risk of recurrence in women with bipolar disorder during pregnancy: prospective study of mood stabilizer discontinuation. Am J Psychiatry 164: 1817-1824.

31. Munk-Olsen T, Laursen TM, Meltzer-Brody S, Mortensen PB, Jones I (2012) Psychiatric disorders with postpartum onset: possible early manifestations of bipolar affective disorders. Archives of General Psychiatry 69: 428-34.

32. Di Florio A, Forty L, Gordon-Smith K, Heron J, Jones L, et al. (2013) Perinatal episodes across the mood disorder spectrum. JAMA Psychiatry 70: $168-175$.

33. Kulkarni J, Hayes, E, Gavrilidis, E. (2012) Hormones and Schizophrenia. Current Opinion Psychiatry 25: 89-95.

34. Riecher-Rossler A, Kulkarni J (2011) Estrogens and Gonadal Function in Schizophrenia and Related Psychoses. Biological Basis of Sex Differences in Psychopharmacology. Berlin Heidelberg Springer Verlag 155-171.

35. Jablensky AV, Morgan V, Zubrick SR, Bower C, Yellachich LA (2005) Pregnancy, delivery, and neonatal complications in a population cohort of women with schizophrenia and major affective disorders. Am J Psychiatry 162: 79-91.

36. Ross LE, McLean LM (2006) Anxiety disorders during pregnancy and the postpartum period: A systematic review. J Clin Psychiatry 67: 1285-1298.

37. Williams KE, Koran LM (1997) Obsessive-compulsive disorder in pregnancy, the puerperium, and the premenstruum. J Clin Psychiatry 58: 330-334.

38. Hinshaw SP, Stier A (2008) Stigma as related to mental disorders. Annu Rev Clin Psychol 4: 367-393.

39. Andersson L, Sundström-Poromaa I, Bixo M, Wulff M, Bondestam K, et al. (2003) Point prevalence of psychiatric disorders during the second trimester of pregnancy: a population-based study. Am J Obstet Gynecol 189: $148-154$

40. Wisner KL, Sit DK, McShea MC, Rizzo DM, Zoretich RA, et al. (2013) Onset timing, thoughts of self-harm, and diagnoses in postpartum women with screen-positive depression findings. JAMA Psychiatry 70: 490-498.

41. Opler LA, Kay SR, Lindenmayer J P, Fiszbein A (1987) The Positive and Negative Syndrome Scale (PANSS) for Schizophrenia. Schizophrenia Bulletin 13: 261-76.
42. Einarson A, Boskovic R (2009) Use and safety of antipsychotic drugs during pregnancy. J Psychiatr Pract 15: 183-192.

43. Robinson GE (2012) Treatment of schizophrenia in pregnancy and postpartum. J Popul Ther Clin Pharmacol 19: e380-386.

44. Seeman MV (2013) Clinical interventions for women with schizophrenia: pregnancy. Acta Psychiatr Scand 127: 12-22.

45. APGAR V, HOLADAY DA, JAMES LS, WEISBROT IM, BERRIEN C (1958) Evaluation of the newborn infant; second report. J Am Med Assoc 168: 1985-1988.

46. Brockington I (2004) Postpartum psychiatric disorders. Lancet 363: 303-310.

47. McCauley-Elsom K, Kulkarni J (2007) Managing psychosis in pregnancy. Aust N Z J Psychiatry 41: 289-292.

48. Spinelli MG (2009) Postpartum psychosis: detection of risk and management. Am J Psychiatry 166: 405-408.

49. Jones I, Chandra PS2, Dazzan P3, Howard LM4 (2014) Bipolar disorder, affective psychosis, and schizophrenia in pregnancy and the post-partum period. Lancet 384: 1789-1799.

50. Newport DJ, Hostetter A, Arnold A, Stowe ZN (2002) The treatment of postpartum depression: minimizing infant exposures. J Clin Psychiatry 63 Suppl 7: 31-44.

51. Chaudron LH, Pies RW (2003) The relationship between postpartum psychosis and bipolar disorder: a review. J Clin Psychiatry 64: 1284-1292.

52. Suppes T, Baldessarini RJ, Faedda GL, Tohen M (1991) Risk of recurrence following discontinuation of lithium treatment in bipolar disorder. Arch Gen Psychiatry 48: 1082-1088.

53. Hatters Friedman S, Sorrentino R (2012) Commentary: postpartum psychosis, infanticide, and insanity--implications for forensic psychiatry. J Am Acad Psychiatry Law 40: 326-332.

54. Dunsis A, Smith GC (1996) Consultation-liaison psychiatry in an obstetric service. Aust N Z J Psychiatry 30: 63-73.

55. Smith A RC, Richters J, Grulich A (2003) Australian Study of Health and Relationships: Australian Research Centre in Sex, Health and Society. La Trobe University.

56. Austin MP, Priest SR (2005) Clinical issues in perinatal mental health: new developments in the detection and treatment of perinatal mood and anxiety disorders. Acta Psychiatr Scand 112: 97-104.

57. Kulkarni J (2014) Women's mental health: Still not a priority, still not good enough. Aust N Z J Psychiatry 48: 701-704.

58. Mezey GC, Bewley S (1997) Domestic violence and pregnancy. Br J Obstet Gynaecol 104: 528-531.

59. Howard LM, Oram S, Galley H, Trevillion K, Feder G (2013) Domestic violence and perinatal mental disorders: a systematic review and metaanalysis. PLoS Med 10: e1001452.

60. Svare JA, Hansen BB, Mølsted-Pedersen L (2001) Perinatal complications in women with gestational diabetes mellitus. Acta Obstet Gynecol Scand 80: 899-904.

61. Nankervis A, Conn J (2013) Gestational diabetes mellitus--negotiating the confusion. Aust Fam Physician 42: 528-531.

62. Shaat N, Groop L (2007) Genetics of gestational diabetes mellitus. Curr Med Chem 14: 569-583.

63. Kulkarni J, Worsley R1, Gilbert H1, Gavrilidis E1, Van Rheenen TE1, et al. (2014) A prospective cohort study of antipsychotic medications in pregnancy: the first 147 pregnancies and 100 one year old babies. PLoS One 9: e94788.

64. Gentile S (2010) Neurodevelopmental effects of prenatal exposure to psychotropic medications. Depress Anxiety 27: 675-686.

65. Reis M1, Källén B (2008) Maternal use of antipsychotics in early pregnancy and delivery outcome. J Clin Psychopharmacol 28: 279-288.

66. Bodén R, Lundgren M, Brandt L, Reutfors J, Kieler H (2012) Antipsychotics during pregnancy: relation to fetal and maternal metabolic effects. Arch Gen Psychiatry 69: 715-721.

67. Corrigan PW. (1998) The impact of stigma on severe mental illness. Cognitive and Behavioral Practice 5: 201-22. 
Citation: Gilbert H, Gurvich C, Kulkarni J (2015) Special Issues for Pregnant Women with Mental Illness. J Nurs Care 4: 280. doi: 10.4172/2167-1168.1000280

Page 9 of 9

68. Kulkarni J, Storch A, Baraniuk A, Gilbert H, Gavrilidis E, et al. (2015) Antipsychotic use in pregnancy. Expert Opin Pharmacother 16: 1335-1345.

69. Coughlin CG, Blackwell KA, Bartley C, Hay M, Yonkers KA, et al. (2015) Obstetric and neonatal outcomes after antipsychotic medication exposure in pregnancy. Obstet Gynecol 125: 1224-1235.

70. Uçok A, Gaebel W (2008) Side effects of atypical antipsychotics: a brief overview. World Psychiatry 7: 58-62.

71. Muench J, Hamer AM (2010) Adverse effects of antipsychotic medications. Am Fam Physician 81: 617-622.

72. Paschetta E, Rosso, G, Maina, G. (2014b) Atypical antipsychotics , maternal and child outcomes: A critical review. OA Women's Health.18: 1-5.

73. Ban L, Gibson JE, West J, Fiaschi L, Sokal R, et al. (2014) Maternal depression, antidepressant prescriptions, and congenital anomaly risk in offspring: a population-based cohort study. BJOG 121: 1471-1481.

74. Khawam EA, Laurencic G, Malone DA Jr (2006) Side effects of antidepressants: an overview. Cleve Clin J Med 73: 351-353, 356-61.

75. Vajda FJ, O'Brien TJ, Hitchcock A, Graham J, Lander C (2003) The Australian registry of anti-epileptic drugs in pregnancy: experience after 30 months. J Clin Neurosci 10: 543-549.

76. Meador KJ, Baker GA, Browning N, Cohen MJ, Bromley RL, et al. (2013) Fetal antiepileptic drug exposure and cognitive outcomes at age 6 years (NEAD study): a prospective observational study. Lancet Neurol 12: 244-252.

77. Campbell E, Kennedy F2, Russell A3, Smithson WH4, Parsons L5, et al. (2014) Malformation risks of antiepileptic drug monotherapies in pregnancy: updated results from the UK and Ireland Epilepsy and Pregnancy Registers. J Neurol Neurosurg Psychiatry 85: 1029-1034.

78. Galbally M, Roberts M, Buist A; Perinatal Psychotropic Review Group (2010) Mood stabilizers in pregnancy: a systematic review. Aust N Z J Psychiatry 44: 967-977.

79. Rothera I, Oates, M (2008) Managing perinatal mental health disorders effectively: identifying the necessary components of service provision and delivery. Psychiatric Bulletin 32:131-3.

80. Galbally M1, Blankley G, Power J, Snellen M (2013) Perinatal mental health services: what are they and why do we need them? Australas Psychiatry 21: 165-170.

81. Dolman C, Jones I, Howard LM (2013) Pre-conception to parenting: a systematic review and meta-synthesis of the qualitative literature on motherhood for women with severe mental illness. Arch Womens Ment Health 16: 173-196.

82. Myors KA, Schmied V, Johnson M, Cleary M (2014) 'My special time': Australian women's experiences of accessing a specialist perinatal and infant mental health service. Health Soc Care Community 22: 268-277.

83. Jenkins M (2015) Women's views about maternity care: How do women conceptualise the process of continuity? . Midwifery 31:25-30.

84. Myors KA, Schmied V, Johnson M, Cleary M (2014) 'My special time': Australian women's experiences of accessing a specialist perinatal and infant mental health service. Health Soc Care Community 22: 268-277.

85. ANMC (2007) A national framework for the development of decisionmaking tools for nursing and midwifery practice. Melbourne, Australia: Nursing and Midwifery Board of Australia. 